\title{
PROTON THERAPY SPECIAL FEATURE: EDITORIAL Proton therapy special feature: introductory editorial
}

\author{
${ }^{1}$ KATHRYN D HELD, ${ }^{2,3}$ ANTONY J LOMAX and ${ }^{4,5}$ ESTHER G C TROOST \\ 'Department of Radiation Oncology, Massachusetts General Hospital/Harvard Medical School, Boston, MA, USA \\ ${ }^{2}$ Center for Proton Therapy, Paul Scherrer Institute, Villigen, Switzerland \\ ${ }^{3}$ Department of Physics, ETH Zürich, Zürich, Switzerland \\ ${ }^{4}$ Department of Radiotherapy and Radiation Oncology, Faculty of Medicine and University Hospital Carl Gustav Carus, Technische \\ Universität Dresden, Dresden, Germany \\ ${ }^{5}$ OncoRay - National Center for Radiation Research in Oncology, Dresden, Germany
}

Address correspondence to:

Kathryn D Held

E-mail: KHELD@mgh.harvard.edu

Antony J Lomax

E-mail: tony.lomax@psi.ch

Esther G C Troost

E-mail: esther.troost@uniklinikum-dresden.de

Proton therapy for cancers of various types is expanding rapidly all around the world with about 90 facilities in operation and approximately 30 under construction, according to the PTCOG website. ${ }^{1}$ Almost 200,000 patients have been treated with protons, and innovative treatment approaches and exciting results from clinical studies are appearing regularly. This $B J R$ special feature highlights advances in proton therapy in the areas of biology, physics and clinical studies, reviewing the latest research and clinical findings and identifying challenges and opportunities for further research and development.

The dedicated issue begins with two thought-provoking Commentaries, one concerning biology and one addressing a physics question. Dini et al. ${ }^{2}$ ask whether protons can be more efficacious in the treatment of cancer stem cells than photons. Recent studies on cancer stem cells show that they are tumorigenic and able to recapitulate the characteristics of the tumour from which they are derived. Hence, it appears that elimination of this sub population is essential to prevent recurrences, but there is evidence that cancer stem cells are inherently resistant to conventional (photon) radiotherapy. The suggestion that protons may be more effective at killing cancer stem cells than photons is exciting, but more biology studies to assess sensitivity of cancer stem cells to protons appear to be needed.

The second Commentary concentrates on the intriguing issue of arc therapy for protons. There is no doubt that the equivalent development in photon therapy (VMAT) has brought substantial advantages and has rapidly become the delivery modality of choice for the treatment of complex cases in conventional therapy. However, there is some controversy in the proton community whether any additional advantage can be gained with rotational approaches, due to concerns about the loss of conformation to the lowto-mid doses. In the commentary by Carabe et al. ${ }^{3}$ they investigate arc proton therapy from both the pure dosimetric, but also biological aspect, concluding that, although dosimetrically there appears to be little advantage to arcing, due to its ability to concentrate more damaging, high LET radiation into the tumour, arc therapy could improve the therapeutic ratio, particularly for brain tumours. An interesting thought which certainly warrants more investigation.

There are five Review articles addressing various biology research issues with protons. Durante and Formenti ${ }^{4}$ consider the important question of whether protons can be more effective than X-rays in combination with immunotherapy. Recent clinical results and pre-clinical experiments point to a benefit for the combined treatment of radiotherapy and immunotherapy in metastatic patients. In this paper, the hypotheses that particles, compared to photons, cause reduced damage to immune cells that are required for an effective immune response, activate different cell death pathways and release cytokine mediators of inflammation.

Three biology Reviews address the issues related to proton relative biological effectiveness (RBE). Friedrich ${ }^{5}$ describes the dependence of the proton RBE on dose in the setting of hypofractionation. Hypofractionation is being used increasingly with protons, and, although the RBE for protons is small, it increases with dose so a better understanding is needed of the implications of this dose-dependence for hypofractionated proton treatments. McNamara et al. ${ }^{6}$ present experimental evidence indicating that proton RBE varies based on technical, tumour and patient factors. The data suggest that using a single scaling factor, i.e. the commonly used RBE of 1.1, to equate the effects of photons and protons across all biological endpoints and doses is too 
simplistic and raises concerns for treatment planning decisions. The article reviews the models that have been developed to better predict RBE variations in tissue based on experimental data and a mechanistic approach. Deycmar et al. ${ }^{7}$ describe the increasing understanding of a differential requirement for the two major DNA double-strand break repair pathways, homologous recombination and non-homologous end joining, in response to proton and photon irradiation. The differential response subsequently influences the mode of cell death and susceptibility of tumour cells with defects in DNA repair machineries to either quality of ionizing radiation. The implications for exploiting these subtle differences in a personalised treatment approach are considered.

The last Review in the biology section serves as a springboard into the physics and clinical papers as Dilmanian et al. ${ }^{8}$ address mini-beam proton therapy, a form of spatially fractionated radiotherapy wherein broad-beam radiation is replaced with segmented mini-beams, either parallel, planar mini-beam arrays generated by a multi-slit collimator or scanned pencil beams that converge laterally at depth to create a uniform dose layer at the tumour. The paper outlines recent studies using computational modelling, phantom experiments, in vitro and in vivo preclinical models and early clinical feasibility assessments that suggest that unique physical and biological attributes of mini-beam proton therapy can be exploited for future clinical benefit.

There are nine Review articles relating to medical physics aspects of proton therapy ranging from overviews of the physical aspects of particles, through to the use of protons to exploit "new biologies" such as high dose rate and spatially fractionated treatments. Whilst Jaekel ${ }^{9}$ reviews the physical advantages of both protons and heavier particles for therapy, Nystrom et al. ${ }^{10}$ and Schreuder and Shamblin ${ }^{11}$ provide "crystal ball" articles, respectively, outlining what advances can be expected in both treatment planning and proton therapy delivery in the next 10 years. From all these articles, it is clear that much clinical potential can still be levered from particles in radiotherapy.

Wohlfahrt and Richter ${ }^{12}$ then provide an overview of the status of pre-treatment imaging. This is an essential part of any highly precise therapy, but given the "stopping" characteristics of charged particles, it is particularly important for particle therapy, an aspect covered comprehensively in this article. The topic of uncertainties in proton therapy is expanded on further in three more Review articles. In the article by Lomax ${ }^{13}$, the specific issue of range uncertainty is tackled from a clinical point of view, and challenges some of the conventional thoughts about this important issue. Without doubt, however, particles are particularly sensitive to changes in anatomy, and adaptive methods to deal with these are subsequently reviewed in the article by Albertini et al. ${ }^{14}$ an exciting area of research with much potential to further improve the precision of particle therapy. Parodi et al. ${ }^{15}$ continue with the theme of range uncertainty, by reviewing the current state of in-vivo imaging of proton range, providing a fascinating view of the myriad techniques under development or being applied to be able to "see" where particles stop in the patient.
The final two Reviews link the physics back to biology. That from Haelg et al. ${ }^{16}$ reviews the recent literature in neutron doses resulting from proton therapy. This was a topic of some controversy a few years ago, but which has largely been forgotten since the introduction of pencil beam scanning. However, as Haelg et al. point out, much remains to be understood about how to measure neutron dose and estimate its biological effect. Finally, Mazal and colleagues ${ }^{17}$ provide a comprehensive review of the role of protons in both FLASH (very high dose rate) and grid (spatially fractionated) therapies. Although in their infancy, both techniques have the potential to revolutionise radiotherapy, and this is an extremely timely article reviewing the status of these two, fast moving topics.

The seven Reviews on the clinical application of proton beam irradiation and on clinical trials cover major tumour sites. Weber and colleagues ${ }^{18}$ summarise the evidence-based application of proton beam irradiation in adult and paediatric patients with a primary brain tumour. Moreover, they highlight the ongoing clinical studies on confirming the role of this radiotherapy technique. Seidensaal et al. ${ }^{19}$ address the role of protons and heavy ions for re-irradiation of tumours originating from the head and neck (including adenoid cystic carcinoma and sarcoma), skull base and brain. Meijer et al. ${ }^{20}$ present an overview of data on reduced toxicity following proton beam therapy for HPVpositive oropharyngeal cancer patients and introduce the modelbased approach used in The Netherlands. The Review by Gjyshi and $\mathrm{Liao}^{21}$, the pioneers in the field of proton beam therapy for non-small cell lung cancer patients, summarises the rationale for and challenges of using this technique to treat thoracic cancers. Moreover, it reviews the current clinical experience and suggests topics for future research. Due to the physical properties, proton beam therapy also seems promising, even although challenging, for tumours originating from the upper abdomen. Raldow et al. ${ }^{22}$ review the clinical outcome and technical challenges of applying protons for hepatocellular carcinoma, cholangiocarcinomas and pancreatic cancers. Thomas and Timmermann ${ }^{23}$ give a concise overview of the role of proton beam therapy in paediatric tumours. Along with high cure rates, the rate of (late) toxicities is reduced using this radiotherapy modality. Finally, perspectives on the National Health System England clinical service and research programme are given by Burnet and colleagues ${ }^{24}$.

In addition to the Review articles highlighted, this dedicated proton therapy issue is enriched by some insightful original research, including a Short communication and 16 proffered Full papers covering many aspects of proton therapy workflow. The original research section starts with a Short communication on the first results of spot-scanning proton therapy with concomitant hyperthermia in large, inoperable sacral chordomas ${ }^{25}$. Whilst Ricotti et al. ${ }^{26}$ report on their long-term experience of patient set-ups for particle therapy at the heavy ion facility in Pavia, Wang et al. ${ }^{27}$ and Rosas et al. ${ }^{28}$ report respectively on treatment planning for localised dose escalation to MRI-guided lesions in the prostate and benchmarking of a commercial system against a clinically validated in-house system. At the heart of treatment planning, however, is the dose calculation, and the importance of this is reflected in three Full papers. Teoh et al. ${ }^{29}$ question the 
general validity of analytical dose calculations for proton therapy in the lung, whilst also identifying patient characteristics where they may be sufficient, whereas Winterhalter et al. ${ }^{30}$ (BJR-D-1900919) point out some important pitfalls in the tuning of Monte Carlo calculations that can lead to quite significant uncertainties despite these algorithms being typically considered as the "gold standard". Guardiola et al. ${ }^{31}$ on the other hand present a detailed validation of Monte Carlo-based dose calculations for proton mini-beam irradiations, underlining the importance of detailed commissioning processes even for Monte Carlo-based dose calculations.

The issue of robustness is also never too far away in proton therapy, and this special feature does not disappoint in this respect either. Three Full papers are dedicated to this subject. In the contributions from Cubillos-Mesías et al. ${ }^{32}$ and Hague et al. ${ }^{33}$ the robustness to set-up, range and anatomical uncertainties are examined in detail, whilst in the contribution from Teoh et al. ${ }^{34}$ an interesting and rare comparison of the robustness of proton therapy compared to VMAT is presented for lung tumours. This latter paper interestingly shows that, using either PTV or robust optimised proton plans, robustness to set-up errors are clinically acceptable for both proton and VMAT plans, and also shows that dose uncertainties also exist in non-proton delivery techniques such as VMAT. The final three physics-focused Full papers cover an equally important part of any radiotherapy treatment technique, namely efficiency and quality assurance. Whilst, Vilches-Freixas et al. ${ }^{35}$ present the first results on the commissioning of a proton therapy machine based on dynamic collimation, Chen et al. ${ }^{36}$ report on their experiences of moving from experimental to alternative methods for patient-specific verifications, with the aim of improving the efficiency of these extremely time-consuming QA processes. Finally, and also on the topic of workflow and treatment delivery efficiency, Mah et al. ${ }^{37}$ describe the efficiency gains that can be obtained in regards to patient throughput, through reducing both energy and between-room switching times.
This special feature concludes with a diverse selection of clinical Full papers. The first of these, by Kountouri et al. ${ }^{38}$ reports on the incidence optic neuropathy induced after spot-scanning proton therapy for tumours originating from the head and neck and skull base. In the cohort, consisting of 216 patient treated between 1999 and 2014, the incidence of optic neuropathy after a mean follow-up of 5.3 years was $6.5 \%$ after delivering a dose of $\geq 45 \mathrm{~Gy}$ (RBE) to the optic nerve(s) and/or optic chiasm. The article by Lawell et al. ${ }^{39}$ highlights the necessity of prospective patient registries, in particular also for paediatric tumours. The "Paediatric Proton/Photon Consortium Registry" is one of these examples, which started data collection in 2012 and since has included approximately 2800 patients. The third clinical Full paper by Mohiuddin and colleagues ${ }^{40}$ deals with early results of spatially fractionated GRID therapy using protons in 10 patients. Half of the patients were metastatic at the time of treatment and half had received prior photon-beam therapy to the identical tumour site. Even although, in $80 \%$ of the patients the tumour regressed or the local symptoms improved, while only resulting in Grade 1-2 side-effects in half of the patients. Finally, Troost et al. ${ }^{41}$ compare different conformal photonand proton therapy techniques for re-irradiating (non-)small cell lung cancer patients. The 154 treatment plans available for 22 patients to be re-treated to individual dose levels taking into account the previous irradiation revealed that IMPT was superior in achieving the highest tumour dose while also decreasing the dose to the organs at risk.

This fascinating $B J R$ special feature on proton therapy covers the latest research and clinical findings in the field, looking at the challenges and opportunities for further research and development in the biological, clinical and physical aspects of proton therapy. We, Kathy, Tony and Esther, thank all contributors to this special issue, including our dedicated reviewers who provided vital expert guidance, and without whom we could not have produced this exciting feature. We hope all readers enjoy this rich, diverse and insightful collection of papers!

\section{REFERENCES}

1. PTCOG website. Available from: https:// www.ptcog.ch/.

2. Dini V, Belli M, Tabocchini MA. Targeting cancer stem cells: protons versus photons. $\mathrm{Br}$ J Radiol 2019; 93: 20190225. doi: https://doi. org/10.1259/bjr.20190225

3. Carabe-Fernandez A, Bertolet-Reina A, Karagounis I, Huynh K, Dale RG. Is there a role for arcing techniques in proton therapy? Br J Radiol 2020; 93: 20190469. doi: https:// doi.org/10.1259/bjr.20190469

4. Durante M, Formenti S. Harnessing radiation to improve immunotherapy: better with particles? Br J Radiol 2019; 93: 20190224. doi: https://doi.org/10.1259/bjr. 20190224
5. Friedrich T. Proton RBE dependence on dose in the setting of hypofractionation. $\mathrm{Br}$ J Radiol 2019; 93: 20190291. doi: https://doi. org/10.1259/bjr.20190291

6. McNamara A, Willers H, Paganetti H. Modelling variable proton relative biological effectiveness for treatment planning. $\mathrm{Br} \mathrm{J}$ Radiol 2019; 93: 20190334. doi: https://doi. org/10.1259/bjr.20190334

7. Deycmar S, Faccin E, Kazimova T, Knobel PA, Telarovic I, Tschanz F, et al. The relative biological effectiveness of proton irradiation in dependence of DNA damage repair. $\mathrm{Br} J$ Radiol 2019; 93: 20190494. doi: https://doi. org/10.1259/bjr.20190494

8. Dilmanian FA, Venkatesulu BP, Sahoo N, Wu X, Nassimi JR, Herchko S, et al. Proton minibeams-a springboard for physics, biology and clinical creativity. Br J Radiol 2020; 93: 20190332. doi: https://doi.org/10. 1259/bjr.20190332

9. Jäkel O. Physical advantages of particles: protons and light ions. Br J Radiol 2019; 93: 20190428. doi: https://doi.org/10.1259/bjr. 20190428

10. Nystrom H, Jensen MF, Nystrom PW. Treatment planning for proton therapy: what is needed in the next 10 years? Br J Radiol 2019; 93: 20190304. doi: https://doi.org/10. 1259/bjr.20190304

11. Schreuder AN, Shamblin J. Proton therapy delivery: what is needed in the next ten years? Br J Radiol 2019; 93: 20190359. doi: https://doi.org/10.1259/bjr.20190359 
12. Wohlfahrt P, Richter C. Status and innovations in pre-treatment CT imaging for proton therapy. Br J Radiol 2019; 93: 20190590. doi: https://doi.org/10.1259/bjr. 20190590

13. Lomax AJ. Myths and realities of range uncertainty. Br J Radiol 2019; 93: 20190582. doi: https://doi.org/10.1259/bjr.20190582

14. Albertini F, Matter M, Nenoff L, Zhang Y, Lomax A. Online daily adaptive proton therapy. Br J Radiol 2019; 93: 20190594. doi: https://doi.org/10.1259/bjr.20190594

15. Parodi K. Latest developments in in-vivo imaging for proton therapy. Br J Radiol 2019; 93: 20190787. doi: https://doi.org/10.1259/ bjr.20190787

16. Hälg RA, Schneider U. Neutron dose and its measurement in proton therapy-current state of knowledge. Br J Radiol 2020; 93 : 20190412. doi: https://doi.org/10.1259/bjr. 20190412

17. Mazal A, Prezado Y, Ares C. Flash and minibeams in radiation therapy: the effect of microstructures on time and space and their potential application to protontherapy. $\mathrm{Br} \mathrm{J}$ Radiol 2019; 93: 20190807. doi: https://doi. org/10.1259/bjr.20190807

18. Weber DC, Lim PS, Tran S, Walser M, Bolsi A, Kliebsch U, et al. Proton therapy for brain tumours in the area of evidence-based medicine. Br J Radiol 2019; 93: 20190237. doi: https://doi.org/10.1259/bjr.20190237

19. Seidensaal K, Harrabi SB, Uhl M, Debus J. Re-Irradiation with protons or heavy ions with focus on head and neck, skull base and brain malignancies. Br J Radiol 2019; 93: 20190516. doi: https://doi.org/10.1259/bjr. 20190516

20. Meijer TWH, Scandurra D, Langendijk JA. Reduced radiation-induced toxicity by using proton therapy for the treatment of oropharyngeal cancer. Br J Radiol 2020; 93: 20190955. doi: https://doi.org/10.1259/bjr. 20190955

21. Gjyshi O, Liao Z. Proton therapy for locally advanced non-small cell lung cancer. $\mathrm{Br} \mathrm{J}$ Radiol 2019; 93: 20190378. doi: https://doi. org/10.1259/bjr.20190378

22. Raldow A, Lamb J, Hong T. Proton beam therapy for tumors of the upper abdomen. $\mathrm{Br}$ J Radiol 2019; 93: 20190226. doi: https://doi. org/10.1259/bjr.20190226

23. Thomas H, Timmermann B. Paediatric proton therapy. Br J Radiol 2019; 93: 20190601. doi: https://doi.org/10.1259/bjr. 20190601

24. Burnet NG, Mackay RI, Smith E, Chadwick AL, Whitfield GA, Thomson DJ, et al. Proton beam therapy: perspectives on the National health service England clinical service and research programme. Br J Radiol 2020; 93:
20190873. doi: https://doi.org/10.1259/bjr. 20190873

25. Tran S, Puric E, Walser M, Poel R, Datta NR, Heuberger J, et al. Early results and volumetric analysis after spot-scanning proton therapy with concomitant hyperthermia in large inoperable sacral chordomas. Br J Radiol 2019; 93: 20180883. doi: https://doi.org/10.1259/bjr.20180883

26. Ricotti R, Pella A, Tagaste B, Elisei G, Fontana G, Bonora M, et al. Long-Time clinical experience in patient setup for several particle therapy clinical indications: management of patient positioning and evaluation of setup reproducibility and stability. Br J Radiol 2019; 93: 20190595. doi: https://doi.org/10.1259/bjr.20190595

27. Wang T, Zhou J, Tian S, Wang Y, Patel P, Jani AB, et al. A planning study of focal dose escalations to multiparametric MRI-defined dominant intraprostatic lesions in prostate proton radiation therapy. Br J Radiol 2020; 93: 20190845. doi: https://doi.org/10.1259/ bjr.20190845

28. Rosas S, Belosi FM, Bizzocchi N, Böhlen T, Zepter S, Morach P, et al. Benchmarking a commercial proton therapy solution: the Paul Scherrer Institut experience. Br J Radiol 2020; 93: 20190920. doi: https://doi.org/10. 1259/bjr.20190920

29. Teoh S, Fiorini F, George B, Vallis KA, Van den Heuvel F, Heuvel FVden. Is an analytical dose engine sufficient for intensity modulated proton therapy in lung cancer? $\mathrm{Br}$ J Radiol 2019; 93: 20190583. doi: https://doi. org/10.1259/bjr.20190583

30. Winterhalter C, Aitkenhead A, Oxley D, Richardson J, Weber DC, MacKay RI, et al. Pitfalls in the beam modelling process of Monte Carlo calculations for proton pencil beam scanning. Br J Radiol 2020; 93 20190919. doi: https://doi.org/10.1259/bjr. 20190919

31. Guardiola C, De Marzi L, Prezado Y. Verification of a Monte Carlo dose calculation engine in proton minibeam radiotherapy in a passive scattering beamline for preclinical trials. Br J Radiol 2020; 93: 20190578. doi: https://doi.org/10.1259/bjr. 20190578

32. Cubillos-Mesías M, Troost EGC, Lohaus F, Agolli L, Rehm M, Richter C, et al. Quantification of plan robustness against different uncertainty sources for classical and anatomical robust optimized treatment plans in head and neck cancer proton therapy. $\mathrm{Br}$ J Radiol 2019; 93: 20190573. doi: https://doi. org/10.1259/bjr.20190573

33. Hague C, Aznar M, Dong L, Fotouhi-Ghiam A, Lee LW, Li T, et al. Inter-fraction robustness of intensity-modulated proton therapy in the post-operative treatment of oropharyngeal and oral cavity squamous cell carcinomas. Br J Radiol 2019; 93: 20190638. doi: https://doi.org/10.1259/bjr. 20190638

34. Teoh S, George B, Fiorini F, Vallis KA, Van den Heuvel F, Heuvel FVden. Assessment of robustness against setup uncertainties using probabilistic scenarios in lung cancer: a comparison of proton with photon therapy. Br J Radiol 2020; 93: 20190584. doi: https://doi.org/10.1259/bjr. 20190584

35. Vilches-Freixas G, Unipan M, Rinaldi I, Martens J, Roijen E, Almeida IP, et al. Beam commissioning of the first compact proton therapy system with spot scanning and dynamic field collimation. Br J Radiol 2019; 93: 20190598. doi: https://doi.org/10.1259/ bjr.20190598

36. Chen M, Yepes P, Hojo Y, Poenisch F, Li Y, Chen J, et al. Transitioning from measurement-based to combined patientspecific quality assurance for intensitymodulated proton therapy. Br J Radiol 2019; 93: 20190669. doi: https://doi.org/10.1259/ bjr.20190669

37. Mah D, Chen CC, Nawaz AO, Galbreath G, Shmulenson R, Lee N, et al. Retrospective analysis of reduced energy switching and room switching times on throughput efficiency of a multi-room proton therapy center. Br J Radiol 2019; 93: 20190820. doi: https://doi.org/10.1259/bjr.20190820

38. Kountouri M, Pica A, Walser M, Albertini F, Bolsi A, Kliebsch U, et al. RadiationInduced optic neuropathy after pencil beam scanning proton therapy for skull-base and head and neck tumours. Br J Radiol 2019; 93: 20190028. doi: https://doi.org/10.1259/bjr. 20190028

39. Lawell MP, Indelicato DJ, Paulino AC, Hartsell W, Laack NN, Ermoian RP, et al. An open invitation to join the pediatric Proton/Photon Consortium registry to standardize data collection in pediatric radiation oncology. Br J Radiol 2019; 93: 20190673. doi: https://doi.org/10.1259/bjr. 20190673

40. Mohiuddin M, Lynch C, Gao M, Hartsell W. Early clinical results of proton spatially fractionated grid radiation therapy (SFGRT). Br J Radiol 2019; 93: 20190572. doi: https:// doi.org/10.1259/bjr.20190572

41. Troost EGC, Wink KCJ, Roelofs E, Simone,CB, Makocki S, Löck S, et al. Photons or protons for reirradiation in (non-)small cell lung cancer: Results of the multicentric ROCOCO in silico study. Br J Radiol 2019; 93: 20190879. doi: https://doi.org/10.1259/ bjr.20190879 